\title{
Les écoulements diphasiques et la sûreté des réacteurs nucléaires
}

\author{
Two-phase flow and nuclear reactor safety
}

\author{
R. Séméria \\ Service des transferts thermiques, CEN, Grenoble
}

Les écoulements diphasiques liquide-gaz ont déjà été abordés dans diverses réunions organisées par la Société hydrotechnique de France, notamment aux Congrès de l'AIRH, en 1976 et en 1979 [1]. Depuis, des livres présentent les divers aspects industriels de ces écoulements [2] [3], ce qui illustre la maturité de ce domaine de la mécanique des fluides. Le génie nucléaire et plus particulièrement les études de sureté des chaudières ont fortement contribué à ce développement [4] [5], non seulement dans la meilleure connaissance des phénomènes, mais aussi dans la mise en œuvre de grands programmes de calcul capables de modéliser le comportement d'une chaudière nucléaire. C'est ce dernier aspect qui est développé à cette session de la SHF.

Le refroidissement du cœur du réacteur à eau sous pression

Les réacteurs à eau ordinaire sous pression constituent l'essentiel du parc nucléaire français. Ils sont décrits dans un livre récemment paru [6].

Un cœur de réacteur, c'est un cylindre de 4 mètres de haut et 3 mètres de diamètre environ dégageant une puissance volumique d'une centaine de kilowatts par litre en régime nominal et de l'ordre de $\mathrm{kW}$ par litre après arrêt. Cette chaleur est dégagée par 40000 barreaux d'oxyde d'uranium gainés de zircaloy $(9,5 \mathrm{~mm}$ de diamètre extérieur) et enlevée par un débit d'eau pressurisé à 150 bars circulant autout des barreaux à une vitesse de 5 mètres par seconde environ.

Trois ou quatre circuits comportant chacun une pompe et un générateur de vapeur évacuent la puissance thermique (fig. 1 ). En régime normal d'eau ne dépasse pas $325^{\circ} \mathrm{C}$, ce qui est inférieur à sa température d'ébullition; seule une ébullition localisée se développe dans la partie la plus chaude du cœur.

Par contre toute diminution de pression amène une vaporisation, d'abord dans les parties les plus chaudes du circuit $\left(325^{\circ} \mathrm{C}\right)$, puis dans les branches froides $\left(285^{\circ} \mathrm{C}\right)$ et le bas du cœur.

Même lorsque la puissance baisse rapidement, il reste à évacuer du cœur la puissance résiduelle (quelques kilowatts par litre); il faut donc assurer un débit d'eau suffisant pour maintenir les gaines de zircaloy très audessous des températures de déformation et d'oxydation rapide $\left(1200^{\circ} \mathrm{C}\right)$.

Des systèmes auxiliaires de refroidissement de secours suppléent aux défaillances du système primaire par injection d'eau ( fig. 2), soit à haute pression, soit à pression intermédiaire (accumulateurs) soit à basse pression.

Il s'agit donc de montrer qu'en toutes circontances ces dispositifs suffisent à assurer le refroidissement du cœur, et qu'ils permettent à l'exploitant de ramener la chaudière dans une configuration où le refroidissement est assuré à long terme.

\section{Les défauts de refroidissement}

Les incidents pouvant conduire à des difficultés de refroidissement du cœur sont classés en quatre catégories de probabilité décroissante :

1. - La catégorie 1 correspond aux transitoires normaux d'exploitation pour lesquels les conditions d'apparition d'écoulements diphasiques sont limitées à des volumes réduits et à des périodes brèves.

2. - La catégorie 2 comprend des transitoires de probabilité, par réacteur et par an, comprise entre 1 et $1 / 100$, et qui, bien sûr, doivent être corrigés par les 


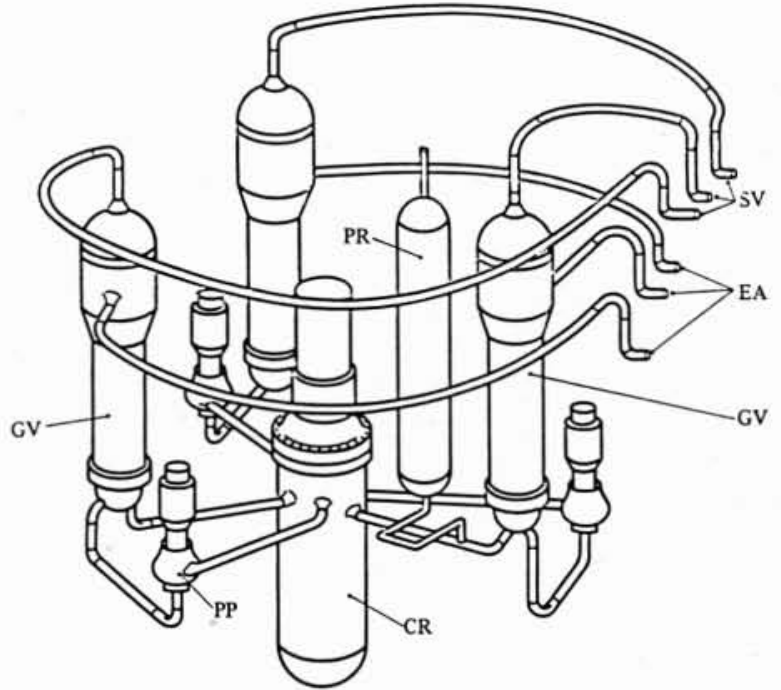

Figure 1. - Configuration générale du circuit primaire d’une chaudière nucléaire à eau sous pression.

La figure montre les trois boucles du circuit primaire d'une tranche de $900 \mathrm{MW}$. Le pressuriseur est branché sur l'une des boucles. Le circuit primaire d'une tranche de $1300 \mathrm{MW}$ cmpote quatre boucles [6].

CR cuve du réacteur; EA eau alimentaire; GV générateur de vapeur; PP pompe primaire; SV sorties vapeur.

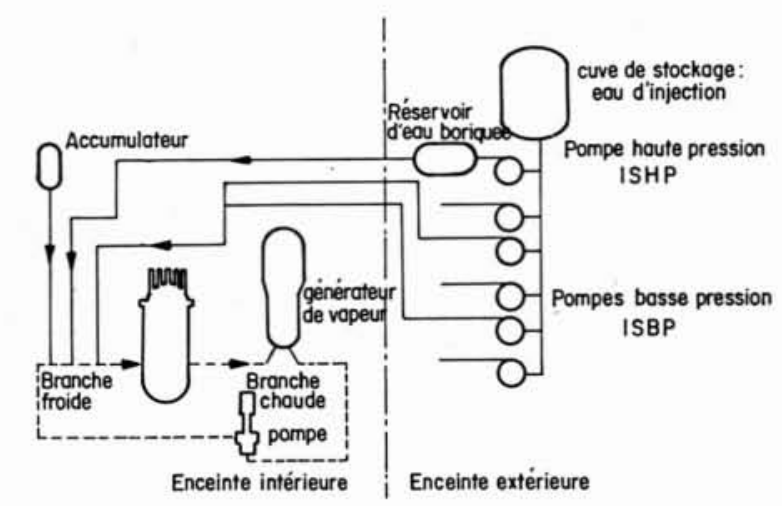

Figure 2. - Systèmes de refroidissement de secours. systèmes de sauvegarde sans dommage pour le réacteur, ni pour l'environnement.

On peut pour certains de ces incidents rencontrer des écoulements diphasiques dans le système primaire: mauvais fonctionnement des générateurs de vapeur, retrait incontrôlé de barres de commande, dépressurisation momentanée, etc.

3. - La catégorie 3 , de probabilité, $10^{-2}$ à $10^{-3}$, comprend des accidents conduisant à un endommagement du cœur. On doit limiter les dégats internes de façon à pouvoir assurer le refroidissement à long terme et ne pas affecter l'environnement autour du site.

C'est dans cette catégorie que se situent les accidents mettant en jeu des écoulements diphasiques plus ou moins généralisés nécessitant des modélisations numériques: petites brèches dans le circuit primaire, ouverture d'une soupape de sureté du pressuriseur,...

4. - En catégorie 4 (probabilité $10^{-4}$ à $10^{-6}$ ), on trouve notamment l'accident dit de référence, de rupture totale d'une tuyauterie du circuit primaire, la rupture d'une tuyauterie d'alimentation principale d'un générateur de vapeur, la rupture de tubes du générateur de vapeur,...

On doit montrer que le cœur reste refroidissable et que l'environnement n'est pas gravement affecté. La modélisation diphasique joue ici un rôle essentiel.

Au-delà, on peut considérer des scénarios très hypothétiques (accidents hors dimensionnement) dont on doit évaluer les conséquences : rupture du circuit primaire par surpression, perte d'alimentation de secours des générateurs de vapeur, défaillances totales diverses.
L'ensemble de ces incidents et accidents nécessitent une modélisation très complète des circuits et composants.

\section{Les besoins de modélisation}

Tout procédé mettant en jeu des systèmes complexes en régime transitoire demande pour être convenablement analysé et exploité une modélisation reposant sur une bonne représentation des comportements globaux des divers constituants du système. C'est ce qui se pratique en génie des procédés [7] et c'est ce qui s'est fait dans les premières tentatives pour l'analyse de la sureté des réacteurs nucléaires. Le programme américain RELAP 4 en est l'aboutissement : il permet au prix d'ajustements empiriques de lois et coefficients de reconstituer les expériences globales de simulation d'accidents et de faire la transposition au cas du réacteur.

Cette approche relativement globale peut donner satisfaction pour un petit nombre de scénarios accidentels, par exemple l'accident de référence. Elle perd de sa crédibilité pour l'étude de séquences accidentelles très diverses, comme par exemple l'ensemble des accidents "petites brèches ", car la vérification des prévisions du calcul sur des expériences globales n'est plus possible pour le nombre quasi infini de combinaisons d'états des divers composants du système.

C'est pourquoi se développent actuellement des programmes de calcul un peu plus analytiques (TRAC aux 
Etats-Unis, CATHARE en France), qui s'appuient sur une modélisation fine telle que la concevrait un mécanicien des fluides.

Chaque modèle physique nécessite une qualification sur des expériences dites analytiques; ensuite des expériences globales permettent de juger de la validité d'ensemble de la modélisation.

En France, Electricité de France et le Commissariat à l'énergie atomique ont développé en commun un programme d'expériences analytiques auquel s'est associé Framatome. Ce programme, commencé en 1974, est réalisé en grande partie. L'effort commun se poursuivra sur un programme d'expériences globales utilisant notamment une installation importante en construction à Grenoble : la boucle BETHSY, qui simule un circuit primaire à l'échelle 1/100 en volume et grandeur réelle en hauteur.

Le développement en commun du programme de calcul CATHARE a rassemblé à Grenoble, depuis septembre 1979, 22 agents de ces organismes. Les conférenciers suivants vous exposeront l'état de leurs travaux.

Conclusions

Cette opération de modélisation d'un réacteur nucléaire en conditions accidentelles à l'aide de programmes de calcul et d'expériences analytiques et globales est exemplaire à plus d'un titre.

- Elle confirme tout d'abord que la compréhension des écoulements diphasiques et de la thermique associée est déjà suffisante pour rendre compte de comportements transitoires de systèmes complexes.

- Les méthodes numériques et les techniques informatiques existantes permettent une exploitation quasi indus- trielle, quoique coûteuse encore, de ces outils de calcul. A brève échéance ils seront utilisés non seulement pour l'analyse mais aussi, après simplification si besoin est, pour l'aide à la décision et à l'intervention humaine lors d'incidents graves en centrales.

Cette méthodologie encore lourde deviendra de plus en plus accessible à des utilisateurs industriels pour la modélisation des procédés; c'est pourquoi il est de l'intérêt des chercheurs et des ingénieurs de suivre de près ces développements.

Références bibliographiques

[1] SÉMÉRIA R. - Progrès récents dans l'étude des systèmes diphasiques liquide-gaz. Evolution des connaissances à l'occasion des études de thermohydrauliques nucléaire. La Houille Blanche n० 6/7, 1979, p. 335.

[2] BERGLES A.E., COLLIER J.G., DELHAYE J.M., MAYINGER F. - Two phase flow and heat transfer in the power and process industries. Hemisphere Publ. Corp. 1981, $707 \mathrm{p}$.

[3] DELHAYE J.M., GIOT M., RIETHMULLER M.C. - Thermohydraulics of Two Phase Systems for Industrial Design and Nuclear Engineering. Hemisphere Publ. Corp. Mac Graw Hill $1981,525 \mathrm{p}$.

[4] SÉMÉRIA R., COURTAUd M., COSTA J. - Nuclear Safety heat Transfer, Keynote lecture. $7^{\text {th }}$ International Heat Transfer Conférence, Munich, sept. 1982.

[5] SÉMÉRIA R., COURTAUD M. - Quelques problèmes de mécanique des fluides et de thermique rencontrés lors des études de sureté des réacteurs Nucléaires. Conférence Générale. 5 Congrès de Mécanique, Marseille sept. 1981.

[6] DREVON G. et alt. - Les réacteurs nucléaires à eau ordinaire. Collection CEA, Serie Synthèses. Eyrolles 1983, $589 \mathrm{p}$.

[7] VILLERMAUX J, et alt. - Vous avez dit Génie des Procédés? Actualité chimique, p. 19, mai 1983. 